Bitte beachten Sie: Aufgrund der Verbreitung des Coronavirus werden zunehmend Veranstaltungen verschoben oder fallen kurzfristig aus. Bitte prüfen Sie vorher, ob ein Kongress stattfindet.

\author{
Die Augenchirurginnen: \\ Phako Power-Wetlab \\ 05.-06. Juni 2020 \\ Düsseldorf, Deutschland \\ www.augenchirurginnen.de
}

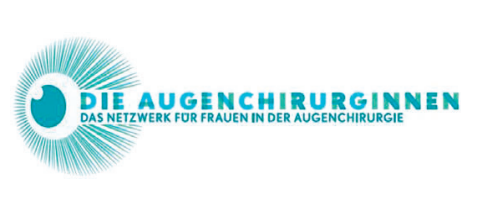

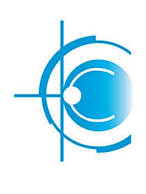

Retinologische Gesellschaft German Retina Society

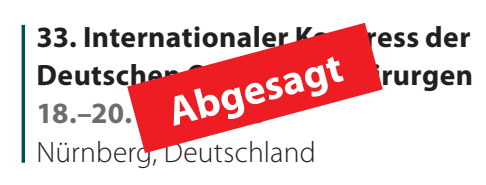

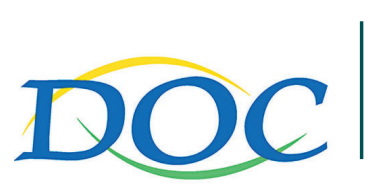

20th EURETINA Congress

01.-04. Oktober 2020

Amsterdam, Niederlande www.euretina.org/amsterdam2020

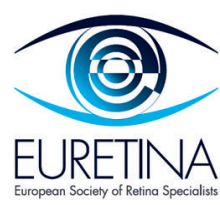

\section{MAI 2020}

Verschoben auf 1.-3. November 2020 61. Jahrestagung der Österreichischen Ophthalmologischen Gesellschaft (ÖOG)

Schladming, Österreich

www.augen.at/veranstaltungen

Verschoben auf 2021

46th Annual Meeting of the European Paediatric Ophthalmological Society (EPOS)

Kopenhagen, Dänemark

www.epos-focus.org

Verschoben auf 14.-16. Dezember 2020 14th Congress of the European Glaucoma Society (EGS)

Brüssel, Belgien

www.egs2020.org

JUNI 2020

Jackstädt-Kurs 2020: Fortbildungskurs der Retinoloaical esellschaft

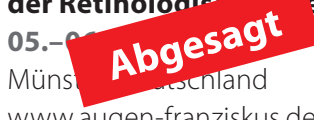

www.augen-franziskus.de

70. Tagung der Vereinigung Norddeutscher ärzte (VNDA)

12.-12 abgesagt

Lübed Abgesand

http://norddeutsche-augenaerzte.de

Webinar

17.-18. Juni 2020

29th International Conference on Insights in Ophthalmology

London, Großbritannien

https://ophthalmology.insightconferences.com
22. Tagung der Bielschowsky-Gesellschaft für Strabologie, Neurn hthalmologie und Pädiatm: : 19.-20. Abgesag

Freiburg Ar Breisgau, Deutschland

www.uniklinik-freiburg.de/bg2020.html

Tübinger Augenärzteabend

24. Juni 2020

Tübingen, Deutschland

www.medizin.uni-tuebingen.de

AUGUST 2020

Sommertagung der Berlin-

Brandenburgischen Augenärztlichen

Gesellschaft (BBAG) 2020

15. August 2020

Berlin, Deutschland

www.bbag-augen.de

Verschoben auf 19.-20. August 2020 27th Edition of European Ophthalmology Congress

London, Großbritannien

https://ophthalmology.euroscicon.com

35. Sulzbacher Ophthalmologischer Dialog (SOD)

26. August 2020

Sulzbach, Deutschland

www.augenklinik-sulzbach.de/aerzte/

aerztliche-fortbildungen

113. SOG Kongress

Annual Congress of the Swiss Society of Ophthalmology

27.-28. August 2020

Ecublens, Schweiz

https://sog-sso2020.congress-imk.ch
4. Retina-Update 29. August 2020 Münster, Deutschland http://retina-update.congresse.de

\section{SEPTEMBER 2020}

28. Jahrestagung der Gesellschaft der Augenärzte Sachsen-Anhalts und Thüringens e.V. (SATh) 04.-05. September 2020 Magdeburg, Deutschland www.sath-augen.de

5th Glaucoma Symposium 04.-05. September 2020

Mainz, Deutschland

https://glaucoma-mainz.de

\section{Frankfurter Imaging Tag (FIT)}

05. September 2020

Frankfurt am Main, Deutschland

www.uni-augenklinik-frankfurt.de/termine/ aerzte

116. Schober-Kurs

07.-11. September 2020

München, Deutschland

http://cms.augeninfo.de

9th International Conference on the Tear Film \& Ocular Surface (TFOS) 2020 09.-12. September 2020

Cernobbio, Italien

ww.tearfilm.org 